\title{
Rush Immunotherapy Using IFN-Gamma for Aspirin in Acute Coronary Syndrome: Case Report
}

Geunwoong Noh ${ }^{1 *}$, Seung Ho Kang ${ }^{2}$, Kyutaek Lee ${ }^{3}$, Woo Jin Kim ${ }^{3}$, Chi Wha Han ${ }^{4}$, Ihl Bohng Choi ${ }^{5}$, Chang Won Ha ${ }^{6}$ and Hyeryeon Noh ${ }^{7}$

${ }^{1}$ Department of Allergy, Allergy and Clinical Immunology Center

${ }^{2}$ Department of Internal Medicine

${ }^{3}$ Department of Laboratory Medicine

${ }^{4}$ Department of Hemato-Oncology

${ }^{5}$ Department of Radiation Oncology

${ }^{6}$ Department of Pathology, Cheju Halla General Hospital, Seoul Korea

${ }^{7}$ Clinical Pharmacy Coordinator, Central Valley Specialty Hospital LA, USA

*Corresponding author: Geunwoong Noh, Department of Allergy, Allergy and Clinical Immunology Center, Cheju Halla General Hospital, Doreongno 65, Jeju-si Jeju Special Self-Governing Province 63127, Korea, Tel: +82-64-740-5064; Fax: +82-64-743-3110; E-mail: admyth@naver.com

Received date: November 13, 2017; Accepted date: November 17, 2017; Published date: November 27, 2017

Copyright: $\odot 2017$ Noh G, et al. This is an open-access article distributed under the terms of the Creative Commons Attribution License, which permits unrestricted use, distribution, and reproduction in any medium, provided the original author and source are credited.

\begin{abstract}
Aspirin plays a key role in the secondary prevention of atherothrombotic events and thrombotic complications after stent implantation and aspirin sensitivity is a limitation for the implantation of bare-metal and drug-eluting coronary stents. Several desensitization protocols have been described. In this report, desensitization by introducing IFN-gamma as an immunomodulatory adjuvant was successful for the patients who required early invasive management and the prompt administration of aspirin but failed the classical desensitization by showing allergic symptoms and signs during desensitization.
\end{abstract}

Keywords: Desensitization; Drug Allergy; IFN-gamma; Aspirin; Immunotherapy

\section{Introduction}

Aspirin plays a key role in the secondary prevention of atherothrombotic events and thrombotic complications after stent implantation [1]. Aspirin sensitivity not only limits patients from benefitting from the long-term use of this antiplatelet agent but is also often a limitation for the implantation of bare-metal and drug-eluting coronary stents.

Three types of aspirin sensitivity have been described: respiratory sensitivity (asthma and/or rhinitis), cutaneous sensitivity (urticarial and/or angioedema), and systemic sensitivity (anaphylactic reaction) $[2,3]$. The prevalence of aspirin-exacerbated respiratory tract disease is approximately $10 \%$, and for aspirin-induced urticaria, the prevalence varies from $0.07 \%$ to $0.2 \%$ in the general population $[3,4]$.

Acetylsalicylic acid desensitization therapy might be an option for aspirin-allergy patients who require coronary stent implantation [5]. Acetylsalicylic acid desensitization therapy involves slowly increasing exposure to oral acetylsalicylic acid to reduce and/or eliminate pharmacologic and presumed immunologic reactions [6]. Several desensitization protocols have been described $[7,8]$.

IFN-gamma has been used for the tolerance induction for food allergies of both IgE-mediated and non-IgE mediated type [9]. IFNgamma was reported to have tolerogenic effects for allergen-specific allergy [10]. During the desensitization for allergenic drugs, nothing could be done if patients showed allergic reactions when exposed to challenging allergenic drugs repetitively, especially in urgent and inevitable situation until now.
In this report, desensitization by introducing IFN-gamma as an immunomodulatory adjuvant was successful for the patients who required early invasive management and the prompt administration of aspirin but failed the classical desensitization by showing allergic symptoms and signs during desensitization.

\section{Case Report}

\section{Patient diagnosis and evaluation of patients}

Case 1: Rush immunotherapy for aspirin desensitization without IFN-gamma

An 82 year-old male patient was admitted to the intensive care unit with a myocardial infarction. The patient had a past history of hypertension, benign prostate hypertrophy, duodenal adenoma and gastrointestinal bleeding. The patient showed chest pain one day before admission. He visited a local clinic and was referred to Cheju Halla General Hospital suspicions of a myocardial infarction. He visited the department of cardiology, internal medicine and was admitted to the intensive care unit with the impression of acute myocardial infarction.

On admission day 2, a cardiac angiography was performed and revealed diffuse eccentric stenosis (90\%) of the proximal left circumflex artery. The right coronary artery was totally occluded with antegrade thrombolysis in myocardial infarction (TIMI) 0 flow and collateral Grade III flow from left anterior descending to the right coronary artery. The echocardiography showed severe hypokinesia with decreased EF $35 \%$ in left coronary artery territory. The electrocardiogram showed normal sinus rhythm and $\mathrm{T}$ wave inversion in V3 to V6 leads. Initial laboratory tests revealed the elevated level of CKMB (48.19 ng/ml, normal range $<5.0 \mathrm{ng} / \mathrm{ml}$ ). The patient was 
diagnosed with non ST elevation myocardial infarction. The patient showed intermediate luminal narrowing of the proximal left circumflex, left anterior descending artery, and collateral formation to the right atrium and chronic total occlusion of the right coronary artery. However, at the dawn of admission day \#3, chest pain was aggravated again. ST segment elevation in EKG was appeared with chest pain. Heparinization was started. Percutaneous cardiac intervention was indicated and administration of aspirin was necessary but the patient had aspirin hypersensitivity and requested consultation to Allergy and Clinical Immunology Center, Cheju Halla General Hospital.

The patients started oral challenge/desensitization for aspirin with protocol of CHH Basic-01 (Table 1). Desensitization was successful up to the dose of $300 \mathrm{mg}$ for $3 \mathrm{~h}$ and at the day $5^{\text {th }}$ of admission, the patient received a percutaneous transcoronary angioplasty (PTCA) with $2^{\text {nd }}$ generation DES stent at proximal circumflex artery of major left coronary artery successfully with medication of aspirin at the dose of $100 \mathrm{mg}$ daily.

\begin{tabular}{|c|c|c|c|}
\hline Time & Dose & IFN-gamma & Cycle \\
\hline 0 & 25 & & \\
\hline 30 & 50 & 0 & 1 cycle \\
\hline 60 & 75 & & \\
\hline 90 & 100 & & \\
\hline 120 & 150 & & \\
\hline 150 & 200 & & \\
\hline 180 & 300 & & \\
\hline
\end{tabular}

Table 1: Aspirin desensitization protocol using IFN-gamma. IFNgamma administration was checked. Repeated count at the same dose is expressed as cycle.

Case 2: Rush immunotherapy for aspirin desensitization using IFNgamma

A 62 year-old male patient was admitted with recently aggravated chest pain. He complained a chest pain for several months. The patient was taking oral medication for hypertension which was diagnosed 1 month before. The patient complained an intermittent chest pain and was referred from the local clinic to the department of cardiology, Cheju Halla General Hospital for further evaluation. The impression was an unstable angina.

Troponin $\mathrm{T}$ and $\mathrm{CKMB}$ were normal and an echocardiogram revealed normal left ventricular function without regional wall motion abnormality. His electrocardiogram showed normal sinus rhythm with RBBB. A coronary angiography was performed. The left coronary angiography showed $60-70 \%$ stenosis in proximal left anterior descending artery and right circumflex artery showed no significant stenosis. We performed stent implantation for proximal left anterior descending segment, after pre-dilation with $2.50 \mathrm{~mm}$ balloon. In the past history, angioedema was developed by taking analgesics (NSAID) after tooth extraction. The patient also had a drug allergy to acetaminophen. The patient showed angioedema and generalized urticarial after taking aspirin for the percutaneous coronary intervention. He was undergoing a planned percutaneous coronary intervention and consultation was requested for the management of aspirin allergy in this situation.

A pharmacologic consultation by Clinical Pharmacy Coordinator at Central Valley Specialty Hospital (Modesto, CA USA) concerning pharmacologic and toxic side effects of the accumulation doses during the desensitization for aspirin, having been given the information of the patients status, especially about the expected effects of IFN-gamma on acute myocardial syndrome was requested.

\section{1) Skin prick test for aspirin}

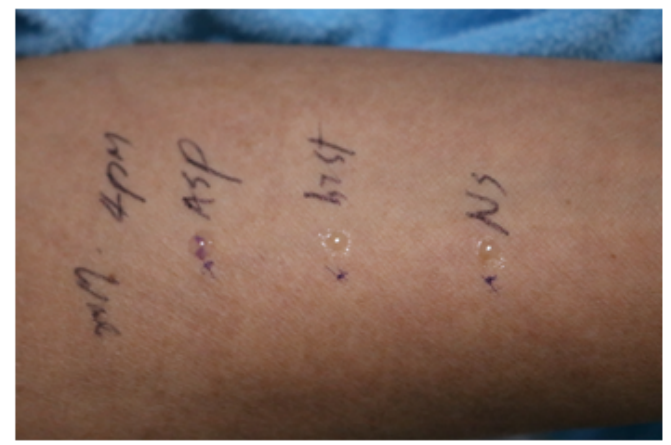

2) Skin test result

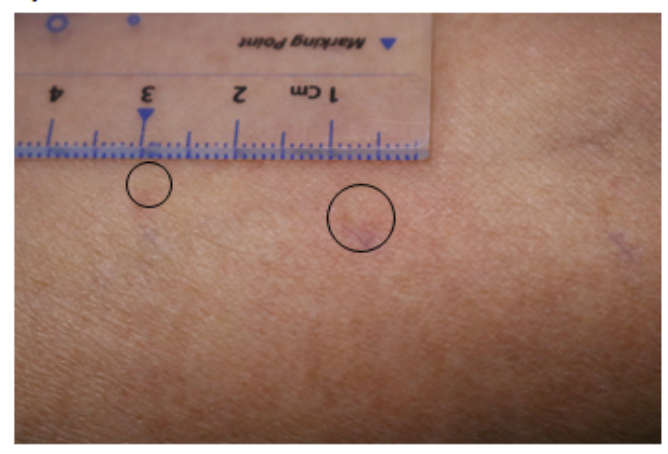

Figure 1: Positive skin prick test result for aspirin. Wheal sizes are 3 $\times 3 \mathrm{~mm}$ for aspirin and $5 \times 5 \mathrm{~mm}$ for histamine (positive control) and $0 \times 0 \mathrm{~mm}$ for normal saline (negative control). ASP: Aspirin; hist: Histamine; NS: Normal Saline.

A skin prick test and intradermal test was performed. Wheal sizes were $3 \times 3 \mathrm{~mm}$ for aspirin, $5 \times 5 \mathrm{~mm}$ for histamine as positive control and $0 \times 0$ for normal saline as negative control (grade II) (Figure 1). Patients received desensitization according to $\mathrm{CHH}$ Protocol Basic 01. However, the patient was itching and showed wheals by taking aspirin at the dose of $50 \mathrm{mg}$ at twice consecutively. Even, allergic reaction was aggravated at the second challenge. According to the classical concept, the desensitization failed and was stopped. However, due to the urgency of patient, IFN-gamma was introduced as an allergentolerogenic immunomodulatory drug. Intermax-gamma (LG Chemistry ${ }^{\circ}$, Seoul Korea) 25 ug was injected subcutaneously. After 15 min, $50 \mathrm{mg}$ of aspirin was re-challenged to the patient for desensitization. Surprisingly, the patient did not show allergic reaction. The impediment during desensitization progress at dose of aspirin 50 mg was resolved (Table 1). Desensitization proceeded according to the protocol, further. The patient showed no more impediments during the remaining desensitization and was successfully desensitized. Patients showed an impediment just once at the dose of $50 \mathrm{mg}$. The resolution 
of the impediment using IFN-gamma was needed only a cycle in this case.

Immediately after aspirin desensitization, the patient received PTCA with stent insertion at left main HTN successfully with using aspirin.

Signed consent forms were obtained from the patients. This treatment was approved by the Institutional Review Board (IRB) of Cheju Halla General Hospital (CHH-IRB-2017-0xx), Jeju-si, Korea.

\section{Discussion}

Hypersensitivity to acetylsalicylic acid (ASA) constitutes a serious problem for patients with coronary artery disease [11]. To ensure these patients get the best therapy, rather than receiving a second choice including coronary artery bypass grafting, the collaboration between allergists and cardiologist is essential. The literature on this issue is currently insufficient and uneven regarding the procedures used.

In the case of an acute coronary syndrome, in patients with STsegment elevation (ACS-STEMI), the primary percutaneous coronary intervention (pPCI) is a safe and effective therapeutic strategy. For this feature, it is mandatory to perform the desensitization procedure in a very short time $(<2 \mathrm{~h})$ to diminish further myocardial damage. The oral dose of aspirin to reach is $150 \mathrm{mg}$ [12]. However, in patients with ACS-STEMI, there is usually not enough time for performing an aspirin desensitization procedure before the percutaneous coronary intervention. A safe choice would be the recommended use of an alternative antiplatelet drug (e.g. clopidogrel, an adenosine diphosphate receptor antagonist) along with a platelet glycoprotein IIb/ IIIa inhibitor (i.e. abciximab, eptifibatide, or tirofiban), as a temporary measure before performing an aspirin desensitization. In any case, in high-risk acute coronary syndrome patients, the desensitization procedure appears to be the best and safest choice, even in those with histories of no severe anaphylactic reactions to ASA/NSAIDs. First of all, practical protocol for patients requiring early invasive management in whom the prompt administration of aspirin is required [1]. The desensitization for aspirin in coronary artery disease is the time consuming success of desensitization. However, limitation of aspirin desensitization is that in patients who failed the desensitization protocol, a new attempt to desensitize was absent in a recent report [13].

IFN-gamma was reported to have tolerogenic effects for both inhalant [14] and food allergens [10]. Moreover, IFN-gamma raised the successful rate and efficacy of desensitization in specific oral tolerance induction for anaphylactic IgE-mediated food allergy [15] and its protocol is well-established [9]. One of mechanism for tolerance induction for allergen-specific allergies was reported as the induction of allergen-specific regulatory $\mathrm{B}$ cells in vivo and in vitro [11].

IFN-gamma was introduced in this report for aspirin desensitization. The principle of overcome of prior dose [16] was applied to overcome the impediment during aspirin desensitization as described in the previous report for food allergy (Figure 2) [15]. In this report, desensitization of aspirin using IFN-gamma was successful as the new therapeutic protocol for the failed desensitization case at least in case of an urgent status. IFN-gamma seems to also have tolerogenic effects on allergenic drug. However, the application of IFN-gamma in drug allergies was different from that in food allergy. IFN-gamma was administrated every challenge in food allergy with interval of at least a day. When the patient showed allergic reaction repetitively at a certain dose, IFN-gamma was administered before the challenge and was not used thereafter when patient did not show allergic reaction after impediment dose. In drug allergies, IFN-gamma was used only at the impediment of desensitization process.

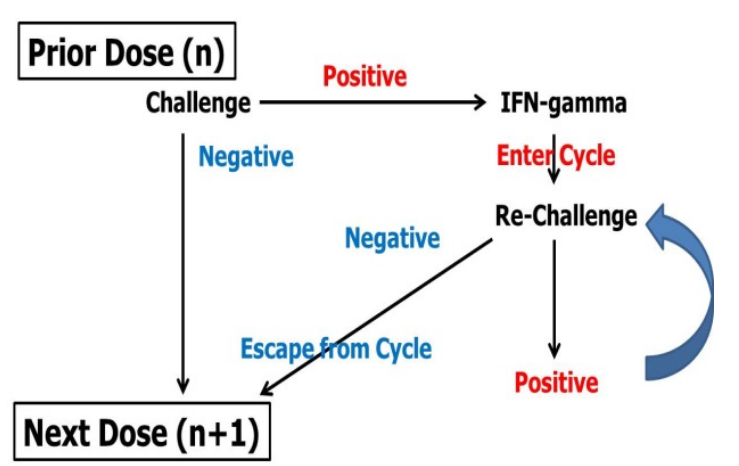

Figure 2: Schematic representation of the resolution of the impediment using IFN-gamma for desensitization of oral aspirin. The principle of the overcoming of prior dose in specific oral tolerance induction for anaphylactic IgE-mediated food allergy was applied for the resolution of the impediment during aspirin desensitization. IFN-gamma was used for the resolution of the impediment during desensitization for aspirin allergy.

The characteristics of this case describe drug allergies for oral medication and the resolution of the impediment using IFN-gamma which needed just a cycle. IFN-gamma seems to be tolerogenic for allergenic drug like being for allergenic food. IFN-gamma may also be usefully applicable to the desensitization for the allergies to many other drugs as a new concept.

\section{Conflicts of Interest}

The authors declare that there is no conflict of interest regarding the publication of this paper.

\section{Acknowledgement}

First of all, I remember Chanyong Noh, Yeonseo Noh and Damseo Noh during writing this manuscript. Also, I appreciate to my wife (ARA) for her excellent support in finishing this work.

\section{References}

1. Rossini R, Angiolillo DJ, Musumeci G, Scuri P, Invernizzi P, et al. (2008) Aspirin desensitization in patients undergoing percutaneous coronary interventions with stent implantation. Am J Cardiol Mar 101: 786-789.

2. Antithrombotic Trialists' Collaboration (2002) Collaborative metaanalysis of randomized trials of antiplatelet therapy for prevention of death, myocardial infarction, and stroke in high risk patients. BMJ 324 : 71-86.

3. Gollapudi RR, Teirstein PS, Stevenson DD, Simon RA (2004) Aspirin sensitivity: implications for patients with coronary artery disease. JAMA 294: 3017-3023.

4. Ramanuja S, Breall J, Kalaria V (2004) Approach to "aspirin allergy" in cardiovascular patients. Circulation 110: e1-e4. 
Citation: Noh G, Kang SH, Lee K, Kim WJ, Han CW, et al. (2017) Rush Immunotherapy Using IFN-Gamma for Aspirin in Acute Coronary Syndrome: Case Report. J Allergy Ther 8: 269. doi:10.4172/2155-6121.1000269

Page 4 of 4

5. McFadden EP, Stabile E, Regar E, Cheneau E, Ong AT, et al. (2004) Late thrombosis in drug-eluting coronary stents after discontinuation of antiplatelet therapy. Lancet 364: 1519-1521.

6. Gollapudi R, Teirstein P, Stevenson D, Simon R (2004) Aspirin sensitivity: Implications for patients with coronary artery disease. JAMA 292 3017-3023.

7. Grzelewska-Rzymowska I, Roznlecki J, Szmidt M (1988) Aspirin "desensitization" in patients with aspirin-induced urticaria and angioedema. Allergol Immuno Pathol 16: 305-308.

8. Schaefer OP, Gore JM (1999) Aspirin sensitivity: the role for aspirin challenge and desensitization in postmyocardial infarction patients. Cardiology 91: 8-13.

9. Lee JH, Noh G, Noh J, Lee S, Choi WS, et al. (2010) Clinical characteristics of oral tolerance induction of IgE-mediated and non-IgEmediated food allergy using interferon gamma. Allergy Asthma Proc 31: e39-e47.

10. Noh G, Lee SS (2003) Effects of IFN-gamma on milk-specific oral tolerance induction for milk allergy in atopic dermatitis: Food-specific oral tolerance induction using IFN-gamma as adjuvant. In: Marone G (Editor). Clinical immunology and allergy in medicine. JGC Editions, Naples, Italy. Pp: 475-496.

11. Cortellini G, Romano A, Santucci A, Barbaud A, Bavbek S, et al. (2017) Clinical approach on challenge and desensitization procedures with aspirin in patients with ischemic heart disease and nonsteroidal antiinflammatory drug hypersensitivity. Allergy 72: 498-506.

12. Mueller C, Patrono C, Valgimigli M, Collet JP, Roffi M (2015) Questions and answers on diagnosis and risk assessment: a companion document of the 2015 ESC Guidelines for the management of acute coronary syndromes in patients presenting without persistent ST segment elevation. Eur Heart J pii: ehv409.

13. Rossini R, Iorio A, Pozzi R, Bianco M, Musumeci G, et al. (2017) Aspirin Desensitization in Patients With Coronary Artery Disease: Results of the Multicenter ADAPTED Registry (Aspirin Desensitization in Patients With Coronary Artery Disease). Circ Cardiovasc Interv 10: e004368.

14. Noh G, Lee KY (2000) Pilot study of IFN-gamma-induced specific hyposensitization for house dust mites in atopic dermatitis: IFN-gammainduced immune deviation as a new therapeutic concept for atopic dermatitis. Cytokine 12: 472-476.

15. Noh G, Lee SS (2009) A pilot study of interferon-gamma-induced specific oral tolerance induction (ISOTI) for immunoglobulin E-mediated anaphylactic food allergy. J Interferon Cytokine Res 29: 667-75.

16. Noh G, Lee JH (2012) Oral tolerance induction for human food allergy. Inflamm Allergy Drug Targets 11: 131-142. 